\title{
SOME EFFECTS OF IMMUNOLOGICAL FACTORS ON GESTATION IN MICE
}

\author{
D. A. JAMES \\ Department of Zoology, Parks Road, Oxford \\ (Received 16th Fanuary 1967)
}

\begin{abstract}
Summary. Some effects of antigenic dissimilarity of mother and conceptus on placental and foetal growth, and on gestation period were investigated. Mothers were immunized against or rendered tolerant to the foreign antigens of the hybrid foetus. Placentae and foetuses were weighed from the 12th day of pregnancy up to immediately before term. Some of the litters were allowed to come to term and recordings were made of the birth weights and the length of the gestation period.

The results indicate that immunological factors have a significant effect on the size of the placenta and on the growth rate of the foetus. Probably because of this the period of gestation was altered. It was concluded that a unique balance is struck between the transfer area of the placenta and the mass of foetal tissue, to the detriment of the foetus if the placenta is unduly small, but to its advantage if a larger placenta is formed.
\end{abstract}

\section{INTRODUCTION}

Hybrid young are heavier at birth than inbred young in litters of the same size and born to mothers of the same strain. The reason for this difference in birth weight must be due either to the genetic constitution of the foetus or the effect of the uterine environment on the foetus, or to a combination of these factors. There are numerous genetic and environmental factors which can influence the size of the placenta and the foetus (see McLaren, 1965a).

The placenta is an important element of the foetal environment. This junction gives rise to unique problems during pregnancy since the invading trophoblast of the conceptus brings paternal transplantation antigens into close contact with maternal tissues (Medawar, 1953). Billington (1964) has shown that antigenic dissimilarity of mother and conceptus can affect placental size in mice, and it has been reported that this effect can be enhanced or diminished by making mothers immune or tolerant to the paternal strain antigens (James, 1965). The placenta is probably a limiting factor of foetal growth and so any change in the size of the placenta may directly affect foetal size. If this is the case, then the period of gestation might also be affected (Wishart \& Hammond, 1933; McLaren \& Michie, 1963).

The following experiment was designed to investigate this aspect of the immunological problem of pregnancy. 


\section{MATERIALS AND METHODS}

Young adult, virgin females were mated at about 90 days of age. They were examined daily for copulation plugs, and the day of finding the plug was designated Day 0 . Three groups of mice were prepared using $\mathrm{C}_{57} \mathrm{BL}$ females mated with $A_{2} G$ males. A fourth group was used as a control when $C_{57} B L$ females were mated with $\mathrm{C}_{57} \mathrm{BL}$ males.

\section{Group 1 (tolerant)}

Mice of Group 1 were rendered tolerant to transplantation antigens of the paternal strain by intravenous injection of spleen cells at approximately $12 \mathrm{hr}$ after birth, using the technique described previously (James, 1965).

\section{Group 2 (immune)}

Mice of Group 2 were immunized against the paternal strain transplantation antigens using either intraperitoneal injections of paternal spleen suspension or two skin grafts. In some cases both these methods were employed together.

\section{Group 3 (control)}

Mice were untreated and mated with $A_{2} G$ males.

\section{Group 4 (control)}

Mice were untreated and mated with $\mathrm{C}_{57} \mathrm{BL}$ males.

Skin grafts were employed to test tolerance and immunity. Grafts were made to a quarter of the females in Groups 1 and 2 as a test sample. Test grafts were made to the right shoulder of the recipients and, as a control graft, the skin from the right shoulder was transferred to the left shoulder. Grafts were not sutured and were dressed with tulle gras and bound with medicated tape.

The mice were inspected on the 5th day after receiving the graft and the test grafts were compared with the control grafts which normally healed quickly.

Using this method, the mean survival time of skin grafts made from $A_{2} G$ males to $\mathrm{C}_{57} \mathrm{BL}$ females was found to be $10 \pm 2$ days. Survival of healthy skin after 20 days for mice of Group 1 was considered to indicate tolerance, whilst rejection before Day 6 in Group 2 mice was considered to confirm immunity.

Mothers were killed on the required day of gestation and the placentae and foetuses were inspected and counted. Litters containing any re-absorbing foetuses were not included in the statistical analysis and, since it has been shown that the size of the litter can affect the individual placental and foetal weights (McLaren \& Michie, 1960), abnormally small litters of four or less were also excluded. Results recorded here were, therefore, taken only from litters containing five to ten healthy foetuses.

The uterus was removed and placed on filter paper dampened with a little normal saline. Each foetus was removed from its membranes and the placenta gently peeled away from the uterus. The damp filter paper was used to blot the placentae and foetuses so that the embryonic membranes could be easily dissected away. The cleaned placentae and foetuses were weighed on a torsion balance to the nearest milligram and were fixed in formol-saline and stored in $70 \%$ alcohol. 
Histological investigation

The placentae were embedded in paraffin wax, sectioned at 5 to $10 \mu$ and stained with 'Haematal 8' and Biebrich Scarlet (Baker, 1962). Some of the sections were stained with Mowry's modification of the Hale reaction (Mowry, 1958).

\section{Gestation length}

Some of the mice in all groups were allowed to come to term undisturbed. As term approached, the mothers were inspected every $6 \mathrm{hr}$. The length of gestation was measured from midday on Day 0 and calculated to the nearest $6 \mathrm{hr}$ when parturition was concluded and the newborns could be weighed. Only litters containing between six and ten young were included in the analysis. Newborn mice were weighed on a torsion balance to the nearest milligram.

\section{RESULTS}

Mice were killed at various times from the 12th day of pregnancy up to immediately before parturition. It proved to be difficult to make confident weighings before Day 12. The results are recorded in Tables 1 and 2, and growth curves are represented by graphs of weight against time (Text-figs. 1 and 2).

\section{Placental growth}

A difference was observed in placental growth between the four groups investigated. In all groups the placenta reached a maximum weight on about the 14th day of pregnancy and then began to decline in weight towards term. This finding is consistent with earlier reports for the mouse (Enzmann, 1935; McLaren, 1965b). It is noticeable, however, that there is a difference in the maximum weight attained by the placentae in the four groups, and that this difference is maintained throughout the latter half of gestation to a greater or lesser degree.

Statistical analysis of variance was carried out to establish the significance of the differences between the groups on the various days. Each point on the graphs (Text-figs. 1 and 2) represents a mean value for 6 to 8 litters, i.e. about fifty individuals. Each point was considered as a sample, and the variance within samples was compared with the variance between samples. In all cases, except on Day 12, the variance ratio was significant at a $0.1 \%$ level. On Day 12 the probability of such variance lay at the $2 \%$ level. The variance between the groups was, therefore, shown to be significantly greater than the variance within the groups for the majority of the results analysed in this way.

Since the variance between the groups was found to be significant, it was decided to compare groups separately for each day investigated. From the graph in Text-fig. 1, groups were compared in descending order on each day so that Group 2 was compared with Group 3, Group 3 with Group 1, and Group 1 with Group 4. A Student's $t$-test was applied to each pair of groups taken. This analysis showed that the immune Group 2 differs significantly from the control Group 3 at all points $(P<0.01)$. The control Group 3 differs significantly from the tolerant Group 1 on all days $(P<0.01)$ except on Days 12 and 15 


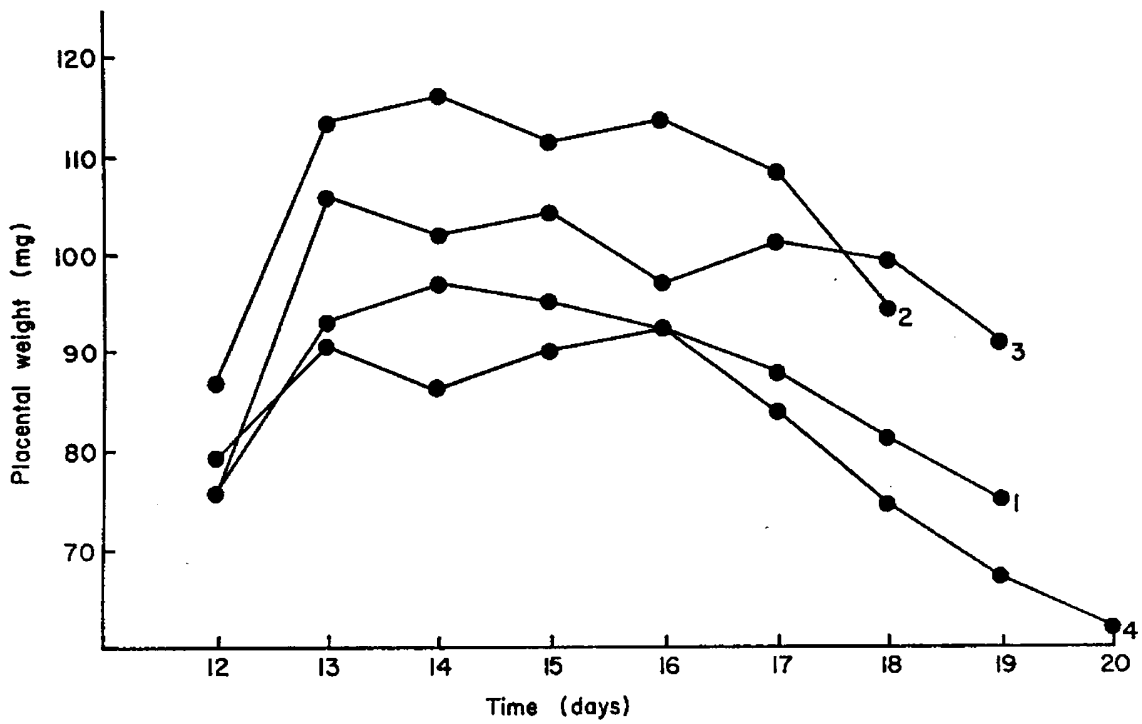

Texr-mo. 1. Placental growth curves during the latter half of gestation in the four groups of mice.

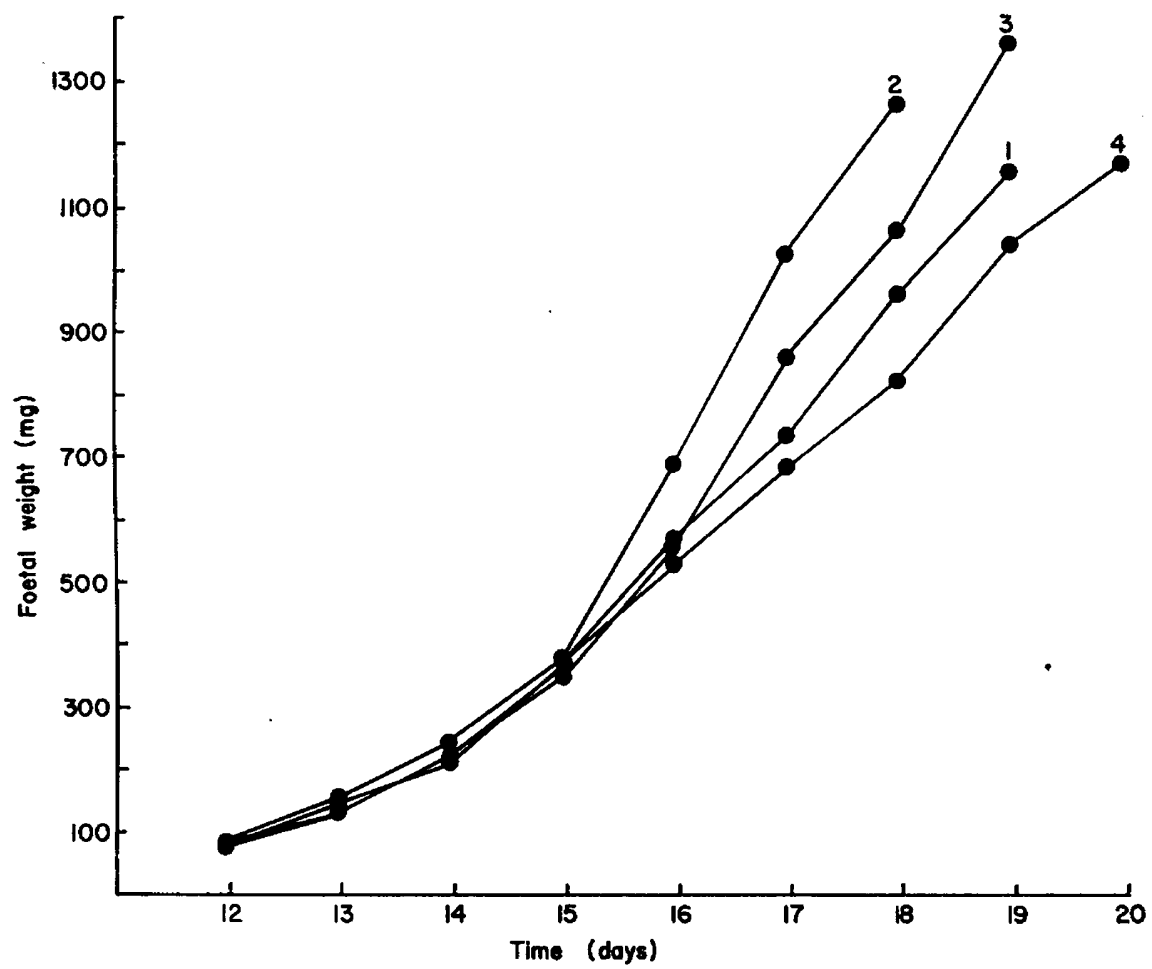

TExT-mi. 2. Foetal growth curves during the latter half of gestation in the four groups of mice. 
where the analysis shows no difference. The tolerant Group 1 differs significantly from the control Group 4 only on Days 14 and 19.

\section{Foetal growth}

The foetal growth curves (Text-fig. 2) are typically sigmoid and for each group the curve is displaced. The foetuses in the immune Group 2 appear to grow at an advanced rate compared with those of the control Group 3, whilst those in tolerant mothers (Group 1) appear to grow at a retarded rate. The growth curve with the smallest slope is that recorded for the inbred foetuses in Group 4 where antigenic dissimilarity of mother and foetus is negligible compared with the other groups.

Statistical analysis was carried out as for the placental weights. The analysis showed that the foetal weights in the immune Group 2 differ significantly from those of the control Group 3 on all days $(P<0.01)$ except on Day 12. Group 3 differs significantly from the tolerant Group 1 only later in gestation on Days 17 and $18(P<0.05)$ and on Day $19(P<0.01)$. Groups 1 and 4 also show a more significant difference approaching term, on Days 16 and 17 $(P<0.05)$ and on Days 18 and $19(P<0.01)$.

The differences between the groups are less marked in the foetal weights than in the placental weights, especially during the earlier stages investigated. This is most noticeable in the record of the tolerant Group 1 where the placental weights are similar to those of Group 4, whilst the foetal weights are similar to those of Group 3.

\section{Relationship between placental and foetal weights}

A positive relation between placental and foetal weight was observed for each day of pregnancy investigated, so that when the placental weight is increased, as in the immunized mothers (Group 2), foetal weight was proportionately increased. This was observed for each group on each day investigated. It was not possible, however, to make a general relationship between placental and foetal growth rates, owing to the peculiar shape of the placental growth curve (Text-fig. 1). For example, in Group 2 the placental weight

\section{EXPLANATION OF PLATE 1}

FIG. 1. Mid-line section of hybrid placenta from Group 1 (tolerant), mother. There appears to be less decidua incorporated in this placenta when compared with the other two groups carrying hybrid placentae (Figs. 3 and 4). The decidual layer shows the same 'density' as in Group 3 placentae. The layer of spongiotrophoblast is not as well defined as in Groups 2 and 3.

Frg. 2. Mid-line section of inbred placenta from Group 4 (untreated), mother. The layer of decidual cells is very thin and the trophoblast giant cells are more prominent than in the hybrid placentae.

Fig. 3. Mid-line section of hybrid placenta from Group 2 (immunized), mother. The cells of the decidua appear to be dispersed and less compact compared to those from Group 3 mothers (see Fig. 4). The trophoblast giant cells are less prominent than in placentae from other groups.

Fig. 4. Mid-line section of hybrid placenta from Group 3 (untreated), mother. The decidual layer is as thick as that shown in Group 2 placentae but appears to be more dense. The trophoblast giant cells are prominent at the periphery.

D, Decidua basalis; ST, Spongiotrophoblast; LT, labyrinth; TGC, trophoblast giant cell. 
PLATE 1

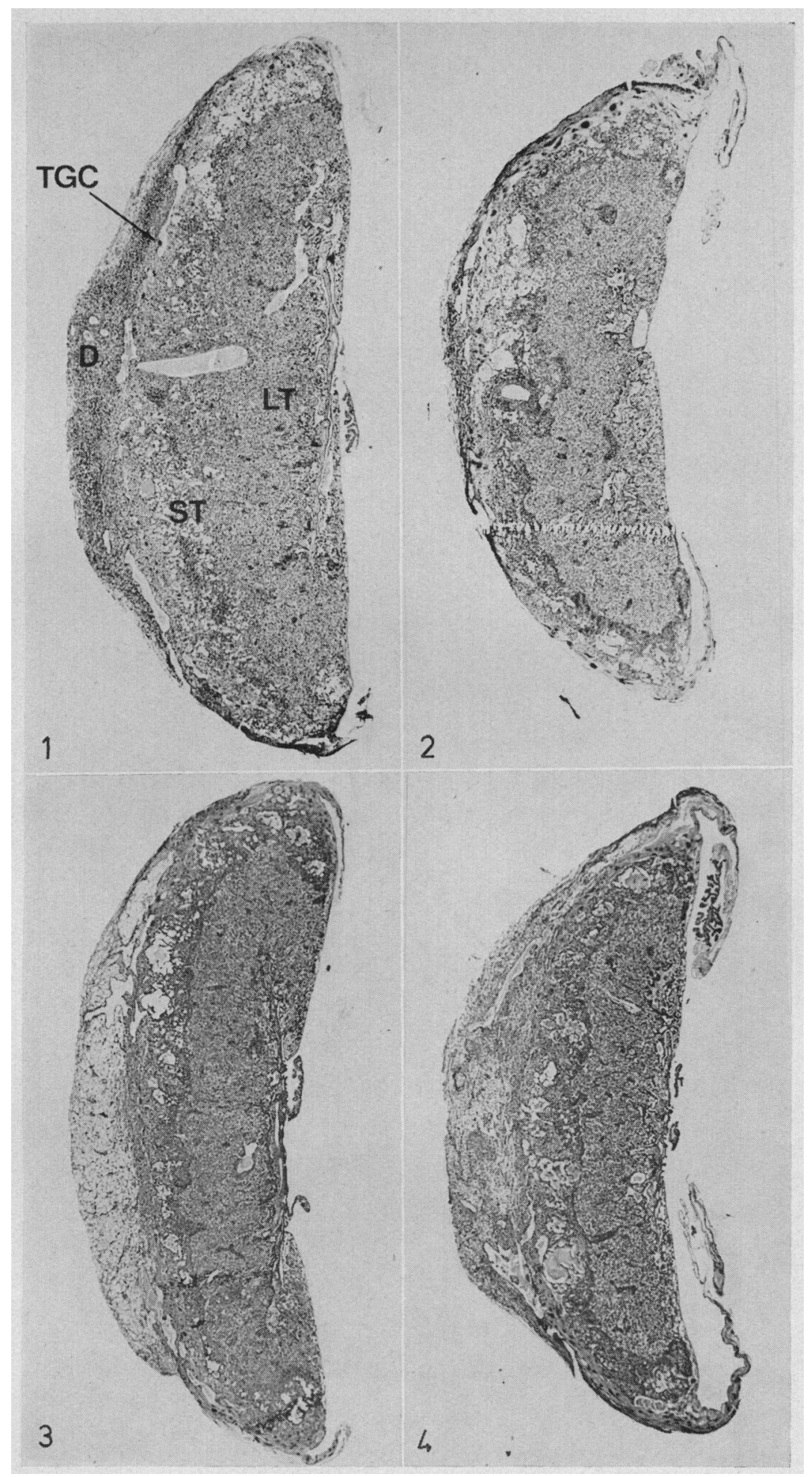

(Facing p. 270) 
on Day 13 is recorded as $113 \mathrm{mg}$, the same as on Day 16. The foetal weight, however, is not the same on these days but follows a normal growth curve. So, for the same placental weight there can be two recordings of foetal weight. Also, for Groups 1 to 3, where all the foetuses are genetically similar, a recorded placental weight of about $90 \mathrm{mg}$ gives very different foetal weights in the three groups, depending upon the degree of immunological dissimilarity existing between mother and foetus.

\section{Histological investigation}

Examination of placental sections collected on Days 13 and 14 of pregnancy revealed some significant structural differences between the placentae of the four groups. There was found to be some variation in the structure and quantity of the decidual layer in the placentae. In placentae collected from immunized mothers the decidua appears to be more abundant and the cells more dispersed in their matrix (see Pl. 1, Fig. 3). The decidual layer in the hybrid placentae in the tolerant and control mothers is not so wide and appears to be more

TABLE 3

SHOWING GESTATION LENGTH IN DAYS, AND BIRTH WEIGHT IN MILLIGRAMS

\begin{tabular}{|c|c|c|c|c|c|c|c|c|c|}
\hline \multirow{2}{*}{ Group } & \multirow{2}{*}{ Mother } & \multirow{2}{*}{ Foetus } & \multirow{2}{*}{$\begin{array}{l}\text { No. of } \\
\text { litters }\end{array}$} & \multirow{2}{*}{$\begin{array}{l}\text { Mean } \\
\text { litter } \\
\text { size }\end{array}$} & \multicolumn{5}{|c|}{ Gestation period in days, and birth weight in $m g$} \\
\hline & & & & & $18 \frac{1}{2}$ & 19 & $19 \frac{1}{2}$ & 20 & $20 \frac{1}{2}$ \\
\hline 1 & Tolerant & $\mathrm{C}_{57} \times \mathrm{A}_{2} \mathrm{G}$ & 9 & 6.7 & - & $\begin{array}{r}1 \\
1118\end{array}$ & $\begin{array}{r}5 \\
1224\end{array}$ & $\begin{array}{r}3 \\
1318\end{array}$ & - \\
\hline 2 & Immune & $\mathrm{C}_{57} \times \mathrm{A}_{2} \mathrm{G}$ & 11 & $8 \cdot 3$ & 9 & 2 & - & - & - \\
\hline 3 & $\begin{array}{l}\text { Control } \\
\text { (hybrid) }\end{array}$ & $\mathrm{C}_{57} \times \mathrm{A}_{2} \mathrm{G}$ & 10 & $7 \cdot 1$ & $\begin{array}{c}1293 \\
- \\
-\end{array}$ & $\begin{array}{r}1427 \\
2 \\
1245\end{array}$ & $\begin{array}{r}- \\
8 \\
1462\end{array}$ & $\begin{array}{l}- \\
-\end{array}$ & $\begin{array}{l}- \\
-\end{array}$ \\
\hline 4 & $\begin{array}{l}\text { Control } \\
\text { (inbred) }\end{array}$ & $\mathrm{C}_{57} \times \mathrm{C}_{57}$ & 10 & $7 \cdot 5$ & - & $\begin{array}{l}- \\
-\end{array}$ & $\begin{array}{l}- \\
-\end{array}$ & $\begin{array}{r}4 \\
1143\end{array}$ & $\begin{array}{r}6 \\
1196\end{array}$ \\
\hline
\end{tabular}

tightly packed (see Pl. 1, Figs. 1 and 4). The inbred placentae collected from control mothers contain only a thin layer of decidua (Pl. 1, Fig. 2).

Trophoblast giant cells are more prominent in the inbred placentae of Group 4 than in the hybrid placentae of the other groups. There is no significant difference in the thickness of the layers of the labyrinthine-trophoblast and spongio-trophoblast between the groups.

Some of the placentae were stained with Mowry's modification of the Hale reaction (Mowry, 1958) in an attempt to investigate the fibrinoid layer around the trophoblast described by Kirby, Billington, Bradbury \& Goldstein (1964). There was found to be no apparent difference in the quantity of fibrinoid in the placentae of the four groups, collected at 13,14 and 17 days of pregnancy.

\section{Length of gestation}

Newborn mice were weighed on a torsion balance to the nearest milligram and were not kept for any further investigation. The results of the birth weights and of gestation period are recorded in Table 3. 


\section{DISCUSSION}

The present results confirm the earlier report that immunological factors have a significant effect on the size of the placenta in mice (James, 1965). It is apparent that this effect occurs early in gestation so that by Day 13 of pregnancy there has developed a significant difference between the placental weights of the four groups used. The present results further confirm that the mouse placenta declines in weight towards term (Enzmann, 1935; McLaren, 1965b).

Foetal growth also appears to be affected by the immunological conditions of this investigation. The foetuses in the immune, tolerant and control Groups 1 to 3 are all of similar genotype $\left(\mathrm{C}_{57} \mathrm{BL} \times \mathrm{A}_{2} \mathrm{G}\right)$ and are all carried by mothers of the same inbred strain $\left(\mathrm{C}_{5}, \mathrm{BL}\right)$, and yet the growth curves for these three groups differ in slope during the latter part of gestation. This indicates that the placenta must be an important limiting factor of foetal growth and that the hybrid genotype alone need not mean an increase in the foetal growth rate.

The observed differences between the four groups are more marked in the placental weights than in the foetal weights, especially in the earlier stages investigated. This suggests that the effect on foetal growth is somewhat secondary, being mediated through the placenta. It is presumably the placenta that is primarily affected.

Histological investigation of the placentae collected during the present experiment suggests that the observed increase in placental weight might be due to the incorporation of more decidua in the formation of the placenta. Whether this might be as a result of more extensive trophoblast invasion is not known. The design of the experiment indicates that the initial cause of this increase in placental weight must be a maternal response to the foreign antigens of the conceptus. Billington (1965) has reported that trophoblast transplanted to extra-uterine sites can invade more extensively when it differs genetically from its host. In utero, however, there is much to suggest that the decidualization of the endometrium might be involved in the suppression of trophoblast invasion (Kirby, 1965). It could be that, during normal pregnancy and when mother and conceptus differ genetically, trophoblast is more invasive and that decidualization is also more pronounced, with the result that a more destructive invasion by the trophoblast is mollified by an increase in decidualization. This hypothesis gains some support from the finding that Grosser's classification of mammals according to the number of layers of maternal and foetal tissue in the placenta can be compared directly with the corresponding degrees of decidual development (Amoroso, 1958), suggesting that the role of the decidua is one of protection rather than nutrition (McLaren, 1965c).

The decidua cannot, however, offer protection to the conceptus for very long after implantation since the trophoblast soon invades the maternal blood vessels. Electron micrographs of these early stages of development show that a layer of extra-cellular material becomes deposited around the trophoblast cells by Day 6 of pregnancy, whilst the conceptus is still embedded in a decidual mass (Potts, 1965). It has been proposed that this layer could act as an antigen barrier between mother and conceptus (Kirby et al., 1964). Park (1958) has reported that experimental trophoblastic emboli elicit little or no inflam- 
matory response in mice. From the evidence available it appears that by the time of trophoblast invasion of the maternal blood vessels there has already been established an effective barrier between the maternal blood and the foetal tissues.

Some of the earlier attempts to demonstrate the presence of histo-compatibility antigens in trophoblast have been unsuccessful (Simmons \& Russell, $1962,1963)$, but more recently it has been reported that immunization of the mother against the antigens of the conceptus can inhibit the development of blastocysts transplanted to the kidney (Simmons \& Russell, 1965; Kirby, Billington \& James, 1966), but not of ectoplacental cones transplanted to the kidney, nor of blastocysts transplanted to the uterus (Kirby et al., 1966). These findings suggest that immunological factors can affect the development of the conceptus in a dramatic way in extra-uterine sites but not in utero. The uterus may act as an immunologically privileged site during the early stages of pregnancy. After Day 6 of pregnancy trophoblast can flourish even when transplanted to different species (Kirby, 1962b; Billington, 1966).

There appear to be two features, therefore, which might combine to give protection to the conceptus during gestation. At implantation, the decidual reaction could be responsible for some immunological protection. During the next 2 days, the trophectoderm differentiates to form trophoblast and a layer of extra-cellular material develops around the trophoblast cells so that an effective antigen barrier exists by the time the trophoblast invades the maternal blood vessels.

It was suggested above that the observed differences between the foetal growth curves may be a secondary effect, mediated through the primary effect on the size of the placenta. It could be, however, that immunological factors might affect foetal morphogenesis directly. Growth and development depend upon the interaction of genetic constitution and environment. In Groups 1 to 3 the genetic constitution is similar but the uterine environment is varied. From the time of fertilization until implantation the environment of the developing egg is the Fallopian tube, and of the blastocyst the uterus. Following implantation, the blastocyst gives rise to an embryo and trophoblast whilst embedded in a decidual mass. The trophoblast invades the sub-mucosa and the definitive placenta becomes formed.

At any of these stages an immune response could have some influence on the process of development. Transplantation immunity involves humoral antibody as well as cell-bound antibody. Humoral antibody can be found in the Fallopian tubes and the uterus during the period of egg development and it has been demonstrated that the egg takes up maternal serum at most stages from the oocyte to the blastocyst (Glass, 1963). Normal development of the blastocyst depends directly on a 'uterine factor' (Kirby, 1962a) and there are many reports of the influence of antibodies on normal development (see Nace, 1955).

The present results do not show any dramatic effects of maternal immunization on the development of the conceptus, but it may be that the increase in placental size as a result of antigenic dissimilarity cannot fully account for the improved growth rate of those foetuses. It would be interesting to investigate 
the rate of bone development in the foetuses of these same four groups throughout the latter part of gestation. Bone development is a useful indicator of foetal maturation, and by comparing the four groups in this way it would be possible to distinguish between an acceleration of morphogenesis or simply an overall increase in foetal mass.

Gestation length is inversely dependent upon litter size (Biggers, Curnow, Finn \& McLaren, 1963). McLaren \& Michie (1963) concluded that the systemic effect of litter size on gestation length reflects the total mass of conceptus tissue. The inter-relations of litter size and the duration of pregnancy on birth weights have also been reported for the rabbit (Wishart \& Hammond, 1933).

The present results confirm that birth weight and duration of pregnancy are related. Further, they show that antigenic dissimilarity of mother and conceptus can affect the length of the gestation period. This effect is probably indirect. Since antigenic dissimilarity affects the size of the placenta and of the foetus, the total mass of the litter in such circumstances would be responsible for stimulating the onset of parturition a little earlier than normal.

It is not yet known why foetal mass affects the length of gestation. It has been suggested that labour is induced when some critical ratio between the demands of the foetus and the ability of the placenta to supply these demands is exceeded (Villee, 1956). The change in this ratio would be determined more by the rapid increase in the demands of the foetus as its mass increases than by the decrease in the ability of the placenta to supply them. The metabolic activity of the placenta decreases as gestation proceeds, but the term placenta is by no means senile (Villee, 1956). The determining factor must involve the mass of the foetus.

It appears that antigenic dissimilarity of mother and foetus so affects the transport efficiency of the placenta that the hybrid foetus is allowed to grow more rapidly, thus attaining a 'term-weight' ratio sooner than normal.

Medawar (1953) has stated that: 'Although there are no factual grounds for supposing that antigenic diversity is anything but an unfortunate consequence of constitutional differences between individuals of a species, yet one is under some obligation to rack one's brains for evidence of any good it might conceivably do. Only thus can antigenic polymorphism be made genetically respectable.' The results of the present series of investigations indicate that there may be some advantage to the conceptus if it differs genetically from its mother. When mother and conceptus are antigenically dissimilar there is some reaction between them which results in the formation of a larger placenta. The foetus is allowed to grow at an increased rate and consequently, either the birth weight is increased or the period of gestation is shortened. The design of this experiment indicates that these effects are not due to heterosis alone but that immunological factors have a significant influence during gestation.

\section{ACKNOWLEDGMENTS}

I would like to thank Dr D. R. S. Kirby for his encouragement throughout this investigation, and I am grateful to Professor F. W. R. Brambell, F.R.s., for his continued interest in this work. 
The present investigation was carried out during the tenure of a PostGraduate Award from the Scientific Research Council.

\section{REFERENCES}

Amoroso, E. C. (1958) Placentation. In: Marshall's Physiology of Reproduction, Vol. 2, Chapter 15. Ed. A. S. Parkes. Longmans, Green, London.

BAKER, J. R. (1962) Experiments on the action of mordants. 2. Aluminium-Haematin. Q. Fl microsc. Sci. 103, 493.

Biggers, J. D., Curnow, R. N., Finn, C. A. \& Malaren, A. (1963) Regulation of the gestation period in mice. F. Reprod. Fert. 6, 125.

Billington, W. D. (1964) Influence of immunological dissimilarity of mother and foetus on size of placenta in mice. Nature, Lond. 202, 317.

Billington, W. D. (1965) The invasiveness of transplanted mouse trophoblast and the influence of immunological factors. F. Reprod. Fert. 10, 343.

Billington, W. D. (1966) Vascular migration of transplanted trophoblast in the Golden hamster. Nature, Lond. 211, 988.

ENZmanN, E. V. (1935) Intrauterine growth of albino mice in normal and in delayed pregnancy. Anat. Rec. 62, 31 .

GLAss, L. E. (1963) Transfer of native and foreign serum antigens to oviducal mouse eggs. Am. Zool. $3,135$.

JAMEs, D. A. (1965) Effects of antigenic dissimilarity between mother and foetus on placental size in mice. Nature, Lond. 205, 613.

KIRBY, D. R. S. (1962a) The influence of the uterine environment on the development of mouse eggs. F. Embryol. exp. Morph. 10, 496.

KIRBY, D. R. S. (1962b) Reciprocal transplantation of blastocysts between rats and mice. Nature, Lond. 194, 785.

KIRBY, D. R. S. (1965) The invasiveness of the trophoblast. Session 3, Symp., Dundee. 'The early conceptus, normal and abnormal'. Livingstone, Edinburgh.

Kirby, D. R. S., Billington, W. D., Bradbury, S. \& Goldstein, D. (1964) Antigen barrier of the mouse placenta. Nature, Lond. 204, 548.

Kirby, D. R. S., Billington, W. D. \& James, D. A. (1966) Transplantation of eggs to the kidney and uterus of immunised mice. Transplantation, 4, No. 6, 713.

McLaren, A. (1965a) Genetic and environmental effects on foetal and placental growth in mice. 7. Reprod. Fert. 9, 79.

MaLaren, A. (1965b) Placental weight loss in late pregnancy. F. Reprod. Fert. 9, 343.

McLaren, A. (1965c) Maternal factors in nidation. Session 1, Symp., Dundee. 'The early conceptus, normal and abnormal'. Livingstone, Edinburgh.

McLaren, A. \& Mrchie, D. (1960) Control of pre-natal growth in mammals. Nature, Lond. 187, 363.

McLaren, A. \& Michie, D. (1963) Nature of the systemic effect of litter size on gestation period in mice. F. Reprod. Fert. 6, 139.

Medawar, P. B. (1953) Some immunological and endocrinological problems raised by the evolution of viviparity in vertebrates. In: Evolution, Symp. Soc. exp. Biol. 7, 320.

Mowry, R. W. (1958) Improved procedure for the staining of acidic polysaccharides by Muller's colloidal-(hydrous)-ferric oxide and its combination with the Feulgen and the P.A.S. reactions. Lab. Invest. 7, 566.

NAce, G. W. (1955) Development in the presence of antibodies. Ann. N.Y. Acad. Sci. 60, 1038.

PARK, W. W. (1958) Experimental trophoblastic embolism of lungs. F. Path. Bact. 75, 257.

Potrs, D. M. (1965) Implantation. Ph.D. thesis, Cambridge University.

Simmons, R. L. \& Russell, P. S. (1962) The antigenicity of the mouse trophoblast. Ann. N.Y. Acad. Sci. 99, 717.

Simmons, R. L. \& Russell, P. S. (1963) The immunological problem of pregnancy. Am. F. Obstet. Gynec. 85, 583.

Simmons, R. L. \& Russelt, P. S. (1965) Histo-compatibility antigens in transplanted mouse eggs. Nature, Lond. 208, 698.

ViLleE, G. A. (1956) Biochemical evidence of ageing in the placenta. In: Ciba Fdn Colloq. Ageing, Vol. 2, "Ageing in transient tissues."

Wishart, J. \& HAMmond, J. (1933) Statistical analysis of the inter-relations of litter size and duration of pregnancy on birth weight in rabbits. 7 . agric. Sci. 23, 463. 